\title{
Design of an Industrial Human-Robot System Through Participative Simulations - Tank Cleaning Case Study
}

\author{
David Bitonneau ${ }^{1,2}$, Théo Moulières-Seban ${ }^{1,3}$, Julie Dumora ${ }^{4}$, Olivier Ly ${ }^{5}$, \\ Jean-François Thibault ${ }^{2}$, Jean-Marc Salotti ${ }^{1}$ and Bernard Claverie ${ }^{1}$
}

\begin{abstract}
Industrials are starting to deploy interactive robots as new solutions to improve workstations. In particular, workstations where human operators may get injured because of repetitive tasks, bad postures or heavy loads are targeted. The introduction of such interactive systems on industrial workstations brings new challenges concerning human cognitive and physical considerations to factories. We followed an approach involving operators, and ergonomics and cognitive engineering skills to improve the introduction of interactive robots in the industry. In this paper, we present the first application of our work on a pyrotechnic tank cleaning workstation. Our approach is illustrated with the design of a solution through several simulation steps involving the workstation's operators. Finally, the design of a prototype based on a teleoperated robot is introduced.
\end{abstract}

\section{INTRODUCTION}

Interactive robotics is being increasingly deployed in the industry, whether through teleoperation, collaborative robots and even exoskeletons [1]. Industrial applications range from material handling and assembly [2], to remote maintenance and inspection [3]. The recent development of the concept of Factory 4.0 increases the industrial demand for these technologies that places humans at the center of automation for better production flexibility. The deployment of interactive robots brings new challenges concerning human cognitive and physical considerations to factories. These human-robot systems also have to satisfy industrial considerations about safety, dependability, productivity, quality and cost.

We faced these issues within Safran in 2012 when the first collaborative robot was introduced to improve work conditions in a foundry to manipulate hot parts of various size and weight. Some important portions of operators' work activity were missed at design stage, resulting in a physical interface not adapted to operators' needs during operations. The system deployment on the workstation lacked integration of operators to qualify it on every work situations. As a consequence some parts could not be handled using the system. Moreover, the training phase was too short for the appropriation of the interactive robot by operators. They were disturbed by virtual guides, failing to understand when they were activated or not. As a result operators found the system too constraining, and global productivity decreased on the workstation. In the end, the system was only used to handle the heaviest parts.

\footnotetext{
${ }^{1}$ Univ. Bordeaux, CNRS, IMS, UMR 5218, 33405 Talence, France

${ }^{2}$ Safran SA, 92130 Issy-les-Moulineaux, France

${ }^{3}$ ArianeGroup, 33167 Saint-Médard-en-Jalles, France

${ }^{4}$ CEA Tech, CTReg Aquitaine, 33600 Pessac, France

${ }^{5}$ LaBRI, Univ. Bordeaux, 33405 Talence, France
}

More generally, this kind of issues with automation in the industry have been reported and analysed [4] [5]. Current design projects lack integration of operators and of their work [6]. Our work aims at enhancing future introduction of interactive robots on workstations, especially in terms of:

- safety and productivity of human-robot interactions;

- complementarity with operators expertise;

- adaptation to operators and industrial variability;

- appropriation of the system by the operators.

To do so, we follow an approach involving operators, and robotics, ergonomics and cognitive engineering skills to design human-robot manufacturing systems. We applied it to a pyrotechnic tank cleaning workstation, for which operations are manual and physically demanding, and for which previous attempts of automation have failed.

\section{FOLLOWED APPROACH}

On top of the usual robotics project team (which usually includes managers, purchasing agents, engineers, maintenance workers and production workers [7]), the followed approach involves ergonomics and cognitive engineering skills, which are often absent from industrial projects. It aims at improving the integration of operators work activity and expertise, and at developing their future activity during the system design [6]. These skills are applied through:

a) Operators activity analysis: (interviews, observations, debriefings [8]) to better understand operators' "realworld" work and the determinants of their activity [6]. By comparing the activity to prescribed procedures, situations of variability can be highlighted as well as how workers cope with them [6]. Results from the activity analysis are incorporated in functional specifications to design a humanrobot manufacturing system that:

- integrates workers expertise (know-how and knowledge integrated by training and experience);

- takes into account determinants (about operators, tools, material, work organization and environment);

- fits the versatility of operators (age, height, health, handicap, expertise) and of their needs;

- leave some leeway for operators to cope with situations of variability [6].

b) Iterative design and participative simulations: to anticipate the transformations of operators' activity and expertise brought by the designed system. Participative simulations involve operators and aim at assessing if the foreseen solution is compatible with their real needs and technical constraints. 


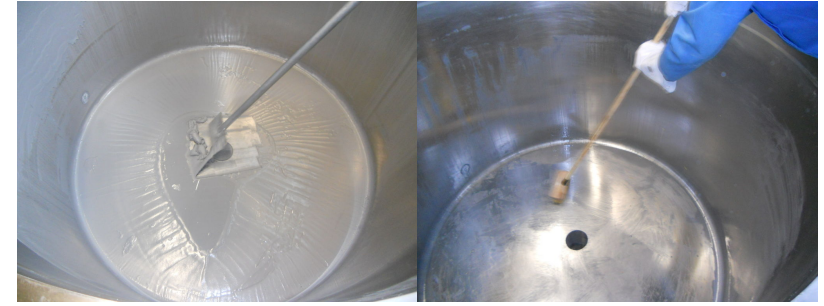

Fig. 1. Current tank scrubbing (on the left) and scratching (on the right).

As a support, models of solutions (which nature can vary from sketches, to cardboard mock-ups, computer models, and prototypes) and "action scenarios" are developed. Action scenarios allow to "play out" situations that operators may have to deal with in the future and reflect the variability of situations emerging from the activity analysis [6]. Iterations of these participative simulations - with increasing representativeness of the real-world work - enable operators to assess if they can manage the wide range of situations they are used to face with the designed interactive robot. These participative simulations also allow to develop operators' future activity [9] and the future process at the same time as designing the technical solution.

\section{TANK CLEANING WORKSTATION ISSUE}

ArianeGroup is specialized in the production of a pyrotechnic polymer. Tanks are soiled during the production process and they have to be cleaned to be reused. Tank's surface is cylindrical, top opened and with a hole on the center of the bottom surface. Tanks are $1 \mathrm{~m}$ high with a diameter of $1.5 \mathrm{~m}$. Two main surfaces have to be cleaned in less than an hour: lateral and bottom. On the bottom surface residue is 1 to $5 \mathrm{~cm}$ thick, while on the lateral surface it is a few millimeters thick. This product being pyrotechnic, its cleaning is a specific process with a required level of safety. Moreover, this substance with high viscosity and stickiness is hard to remove.

Currently cleaning operations are done manually (Figure 1) with four main stages: scrubbing tank's surface to remove most of the substance, scratching to unstick residues, evacuation through the tank's hole and a finishing stage for full cleanliness. They require respectively four types of tools: scrapers, scrub brush with abrasive cleaning pads, squeegee and rags. Most of the polymer has to be collected uncontaminated to be recycled. A plasticizer is used during scratching to liquefy the polymer and facilitate cleaning. These operations are physically demanding for operators. Moreover, they often have to clean several tanks in a row. There is a need to reduce physical load and enhance postures on this workstation.

Studies have been made for years within the company to improve the process of cleaning tanks. Numerous solutions have been tried: solvent based, hydrodynamic and mechanical automation. Currently no satisfying solution has been found to improve operations and work conditions. Previously studied solution were not adopted because either too toxic, making the process more complex, or finally increasing operation time.

\section{RELATED WORK}

\section{A. Cleaning robots and technologies}

Harrington [10] listed three main industrial cleaning technologies: chemical, mechanical and hydrodynamic. Following cleaning systems use combination of these technologies.

Water-based industrial cleaning machines are commercialized for opened tanks [11]. However, such solutions are excluded in our application since the water would contaminate the substance that has to be preserved to be recycled.

The oil and gas industries have related issues of inspection and cleaning of storage tanks containing inflammable products. Shukla et al. [12] review pipe and tank inspection robots in onshore oil-gas industry. Most robots designed for tank inspection are mobile and wall climbing. Fewer cleaning robots have been proposed in the literature for this industry. An oil tank sludge cleaning robot was created to remove $20 \mathrm{~cm}$ thick sludge from the bottom of a $5000 \mathrm{~m}^{3}$ tank [13]. The robot is mobile with high-pressure water jet and a mechanical shovel.

Other cleaning robots include robots for farms, houses [14], building, stores and pipe [15] cleaning. In particular, robots have been designed to clean floors, walls, stairs and windows [16]. All of them are mobile robots.

While mobile robots might be adapted to clean vast surfaces, they are not adapted to clean more little tanks as we have, with bottom and lateral surfaces covered with a highly viscous and sticky product, in less than an hour.

Although this benchmark highlights existing cleaning solutions in related domains, none of them can be adapted to our specific problem.

\section{B. Guiding interactive robots introduction in the industry}

Human-robot interaction has been a major research field for years [17]. Performance and safety issues have received special attention, whether for proximate or remote interaction. Notably, research on telerobotics has tackled these issues for remote operations with the study of control laws to improve stability and transparency of these systems [18]. Safety and dependability aspects of human-robot physical collaboration have been heavily studied recently ([19], [20]), leading to the definition of new standards for the industry (ISO 10218-2 [21] and ISO/TS 15066 [22]).

Work has been done on the assessment of human-robot collaboration acceptability [23] and biomechanical impact [24], using computer simulations.

Yet many ergonomics aspects (such as work determinants, operators expertise and regulations of variability in real work situations, and the development of operators' future activity) are not integrated in these initiatives. Ergonomists have demonstrated the benefits of including these aspects in classical design projects [6]. An approach including these considerations could benefit to the successful introduction of interactive robot systems on industrial workstations. 
TABLE I

MAIN WORK DETERMINANTS OF THE CURRENT TANK CLEANING ACTIVITY

\begin{tabular}{ll} 
Category & Main determinants \\
\hline Operator & (O1) Strength \\
& (O2) Height \\
& (O3) Expertise \\
& (O4) Health and fatigue \\
\hline Tools & (T1) Material \\
& (T2) Length \\
& (T3) Shape \\
& (T4) Dirtiness \\
\hline Material & (M1) Product pyrotechnic sensitivity \\
& (M2) Product viscosity and tack \\
& (M3) Product quantity \\
& (M4) Tank's surface shape \\
& (M5) Tank's surface defect \\
& (M6) Tank's surface reflection \\
& (M7) Product color \\
\hline Work organization & (W1) Production rate \\
& (W2) Night work \\
\hline Environment & (E1) Light \\
& (E2) Temperature and hygrometry
\end{tabular}

\section{DESIGN OF A SOLUTION THROUGH PARTICIPATIVE SIMULATIONS}

\section{A. Operators' activity analysis}

Operators' tank cleaning activity analysis consisted in [8]:

- Interviews: after the study of prescribed procedures, to understand how operators performed the tasks and to collect unusual situations (such as failure context and incidents).

- Observations: to collect and analyse operators' activity (in particular work determinants and workers' expertise) during operations.

- Debriefings: to discuss, validate and enrich with operators the results from the analysis.

Tank cleaning being pyrotechnic, restrictions prevented the direct observation of operators' work activity during operations. Hence, cameras were placed on the workstation for two weeks to collect and analyse operators' activity with their consent.

As a results of our activity analysis we identified the main tank cleaning work determinants. They are classified in Table I, following categories inspired from the $5 \mathrm{M}$ model [25]. Some determinants - such as operators height, product viscosity and tack, and environment light - are the source of variability during operations. Others - such as operators expertise, and tools length and shape - give operators some leeway to cope with this variability.

The operator's activity not only consists in following procedures, it also lies in the regulations (more or less conscious) of variability while performing the task. The analysis also allows to highlight, for each task and subtask, the related determinants and operator's activity. The results from this analysis for tank cleaning operations are summarized in Table II. This table illustrates the variability of situations and, thus, the complexity of operator's activity. Yet, it is not a comprehensive model of the real-world work. For instance, night work influences operator's fatigue, and environment temperature and hygrometry have an impact on product pyrotechnic sensitivity and on operator's fatigue. Ergonomics and cognitive engineering skills are essential to take into account determinants and operator's activity during the following design and simulation iterations to create a system that can cope with variability.

\section{B. Proposed solution}

Our activity analysis revealed that operators adapt their postures, gestures, efforts and choose the proper tool depending on their perception of product's reaction during cleaning. Operators also have a strong expertise to assess the cleaning quality level when only few residues are remaining. Indeed, using the "white cloth test", the residues can be hard to differentiate from the thin layer of plasticizer left on the surface at the end of cleaning. Besides, operators' experience and knowledge of the hazard of the substance (varying depending on the chemical composition, and environment's temperature and hygrometry) is essential to control the safety of operations. Such expertise highlights that operators have to be kept in the loop.

Robot remote control was proposed to take operators away from the hazard during the operations on pyrotechnic product. The roboticist, with the support of operators, made an assumption that the first cleaning steps could be automated to remove most of the residues efficiently. Hence, the proposed interaction ranges from teleoperation when operators need a direct control, to supervisory control for tasks that can be automated [26]. The supervisor role would mainly consist in monitoring and controlling the overall tank cleaning operations through an interface with cameras, ensuring the quality inspection and managing the process security [27]. The system autonomy would be limited and the operators would adjust process parameters or switch to teleoperation when needed.

Several mechanical architectures were considered for the slave robot: gantry robot, SCARA robot, serial articulated robot arm and parallel robot. To face the variability of situations described in the previous section, a 6-axis articulated robot arm was chosen to allow the greatest variety of motions. Robot dimensions and mobility were precised using computer simulations on a simplified model of the workstation. They were determined by the need to reach the whole tank surface, and to fit in the existing workplace. In addition, the various contexts of use were considered: normal (guarantee tank cleaning operation), transitory (facilitate robot's cleaning and maintenance), and failure (anticipate robot removal in case of failure and allow manual cleaning operation without robot obstruction). The tank being top opened, we proposed the robot to be ceiling mounted above it. Due to the workstation geometry, the robot was put on linear axes to allow tank positioning below the robot, and to park the robot for maintenance, cleaning and in case of 
TABLE II

CURRENT TANK CLEANING TASKS, ASSOCIATED DETERMINANTS AND CORRESPONDING OPERATOR'S ACTIVITY

\begin{tabular}{lll} 
Tasks & Current determinants & Operator's current activity \\
\hline Scraper selecting and changing & $(\mathrm{O} 2-3, \mathrm{~T} 1-3, \mathrm{M} 1-4, \mathrm{E} 2)$ & Choosing and grabbing appropriate scrapper \\
\hline Scrubbing & $(\mathrm{O} 1-4, \mathrm{~T} 2-3, \mathrm{M} 1-4, \mathrm{~W} 1, \mathrm{E} 2)$ & Adapting scraper orientation, speed, acceleration, force and penetration \\
\hline Spraying plasticizer and scratching & $(\mathrm{O} 1-4, \mathrm{~T} 2-4, \mathrm{M} 2-4, \mathrm{~W} 1)$ & Adapting brushing speed, acceleration and force \\
\hline Mixed polymer/plasticizer evacuation & $(\mathrm{O} 2-3, \mathrm{~T} 2-3, \mathrm{M}-4, \mathrm{~W} 1)$ & Adapting trajectories and strategies \\
\hline Finishing with rags & $(\mathrm{O} 3-4, \mathrm{~T} 3-4, \mathrm{M} 2-5, \mathrm{~W} 1)$ & Adapting cleaning speed, acceleration and force \\
\hline Rag changing & $(\mathrm{O} 3, \mathrm{~T} 4)$ & Deciding according to cleaning effectiveness \\
\hline Quality inspection & $(\mathrm{O} 3-4, \mathrm{M} 5-\mathrm{M} 7, \mathrm{~W} 1-2, \mathrm{E} 1)$ & Visual or tactile inspection
\end{tabular}

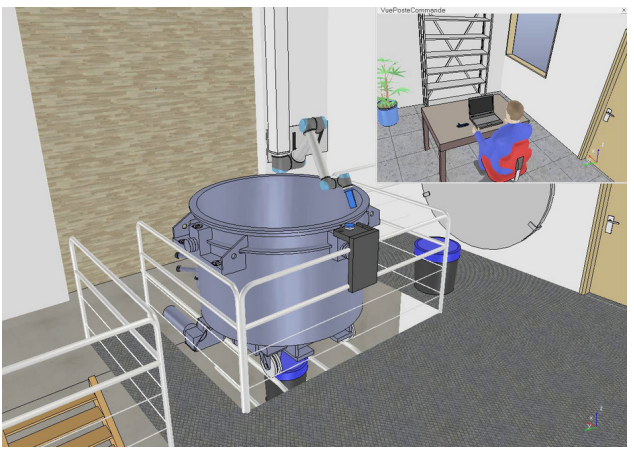

Fig. 2. Computer model of the robot system in the workstation, with an illustration of control room.

failure. The computer model of the solution implemented on V-REP [28] is shown on Fig. 2.

Such a remote controlled robot would introduce new determinants in the future human-robot activity, such as:

(R1) Control system stability and robustness [29].

(R2) Automatic cycles effectiveness and efficiency [30].

(R3) Information displayed for situation awareness [31].

(R4) Teleoperation transparency and stability [18].

The introduction of this system would also transform operators' activity. The foreseen transformations and the impact of new determinants are illustrated in Table III. Major changes can be noticed:

- Operators' strength, height, health and fatigue have a reduced impact on the activity.

- Tool's length becomes transparent for operator activity.

- Operators' expertise is still valued through teleoperation and supervisory control.

- For tasks that can be automated, robot performs automatic cycles and the operator has a supervisor role.

We can also notice that the technical system (robot, programs, control, sensors, tools, interface) designed will have a strong impact on the future safety, quality and productivity of operations. Human and robot will form a team to satisfy these requirements. Hence, the technical system must be adapted to operators, to the task and to its environment. To assess the proposed solution and such transformations, participative simulations are conducted.

\section{Basic participative simulation of the solution}

Simplified automatic cleaning operations from the tank arrival to its depart were implemented in the computer model.
Simulations were conducted with 9 operators, divided in 3 groups, in order to get their assessment of the proposed solution based on their needs and their expertise on the work. First, they were briefly explained the purpose of the simulation and the proposed solution. Then, the simulation was played out. Even though operators could not interact with the virtual model, they were explained their supervisor role when the robot would perform automatic cycles, and that some operations could be teleoperated. The proposed system and activity was then discussed with operators to answer their questions and collect their feedbacks. Finally, we played out and discussed the simulation a second time to collect enlightened feedbacks.

The following subjects were addressed with operators during discussions:

- Missing parts in the simulated activity.

- Ability to deal with every usual situations with the robot.

- Existence of unusual events that could not be handled.

- Willingness to work with the system.

- Advantages and drawbacks of the proposed activity compared to the current one.

- Suggestions to improve the system.

As an example, following qualitative results emerged from discussions with operators:

- Sometimes, crystals of chemical compound remain on the top of the lateral surface. They are harder to remove than the polymer. The system must allow to perform their cleaning.

- Some operations are not within the scope of the system (such as cleaning the external surface of tanks when needed, positioning bins under the tank and cleaning tools). The system must leave leeway for operators to perform these tasks.

- Operators insisted that the system should be simple to use and maintain.

- Operators welcomed that the solution would place them in a safe location and relieve them from physically demanding operation, but they warned about the job interest.

\section{Participative simulation of human-robot interaction}

We then focussed on the study of the system interaction with operators. The previous computer model was improved to allow human interaction with the virtual system. For 
TABLE III

PROPOSED HUMAN-ROBOT TANK CLEANING ACTIVITY WITH UPDATED DETERMINANTS

\begin{tabular}{ll} 
Tasks & Updated determinants \\
\hline Scraper changing & $(\mathrm{O} 3, \mathrm{~T} 1, \mathrm{~T} 3, \mathrm{M} 1-4, \mathrm{E} 2, \mathrm{R} 2-3$ \\
\hline Scrubbing & $(\mathrm{O} 3, \mathrm{~T} 3, \mathrm{M} 1-4, \mathrm{~W} 1, \mathrm{E} 2$, \\
\hline Spraying plasticizer and scratching & $(\mathrm{O} 3, \mathrm{~T} 3-4, \mathrm{M} 2-4, \mathrm{~W} 1, \mathrm{R} 1-4$ \\
\hline Polymer/plasticizer evacuation & $(\mathrm{O} 3, \mathrm{~T} 3, \mathrm{M} 3-4, \mathrm{~W} 1, \mathrm{R} 1-4)$ \\
\hline Finishing with rags & $(\mathrm{O} 3, \mathrm{~T} 3-4, \mathrm{M} 2-5, \mathrm{~W} 1, \mathrm{R} 3-4$ \\
\hline Rag changing & $(\mathrm{O} 3, \mathrm{~T} 4, \mathrm{R} 2-3)$ \\
\hline Quality inspection & \\
& \\
\hline
\end{tabular}

Fig. 3. Simulation setup on the interactive computer model: an operator is using the joystick to control the virtual robot through virtual camera views.

teleoperation, a 2-axis joystick was implemented to move the tools in a tangent direction to tank's surface. The tank being cylindrical, moving the joystick up and down would move the tool along a radius, and moving left and right would move the tool along an horizontal circular arc. Tool's orientation was constrained to face tank's axis of revolution. A joystick button was used to control when the tool touches the surface. A simple graphical user interface was designed for the supervisory control. V-REP virtual cameras were used to give visual feedback on the remotely performed virtual task. The experimental setup for participative simulation is shown in Fig. 3.

The simulations were conducted with 8 tank cleaning operators, one at a time. First, each operator was briefly explained the purpose of the simulation, the proposed solution and the controls. The simulation consisted in playing a supervisory control (virtual robot performs automatic cycles and operator monitors operations through virtual cameras), and then teleoperation. For teleoperation, a training step allowed operators to get familiar with the joystick and simulated cleaning process before playing the task. Finally, the simulation was discussed with each operator. In addition to the subjects presented in the previous section, the usability of the system and visual feedbacks were discussed.

Some of the main results are summarized here:

- Information on robot's state is missing (in particular when it is ready for teleoperation).

- For both supervisory control and teleoperation, operators were satisfied with virtual cameras vision for the
Proposed human-robot activity Human chooses the appropriate scrapper, robot performs automatic change Robot performs automatic cycles, human adjusts parameters if needed Robot performs automatic cycles, human switches to teleoperation if needed Robot performs automatic cycles, human switches to teleoperation if needed Human teleoperates the robot to clean residues Human decides to change rag, robot performs automatic change Human inspects through system visual or force feedbacks

lateral surface cleaning.

- Many operators were disturbed by camera views during bottom cleaning operations, especially near the hole. This is due to cameras being placed on the robot and robot posture varying during motion.

- The field of view of the close task view should be larger.

- Operators warned that the product will stick on the scraper as operations are performed. The system should allow operators to interrupt operations to command scraper's cleaning.

- Although the joystick allowed to perform the simple tasks simulated, operators warned that it might not be sufficient to deal with every situations under real conditions.

While the use of a 2-axis joystick indeed limits the variety of movements allowed by a 6-axis robot, it enabled operators to assess how it would affect real tank cleaning operations. It also allowed to give operators first insights into robot teleoperation and to assess their acceptation.

\section{PROTOTYPE FOR REALISTIC PARTICIPATIVE SIMULATIONS}

\section{A. Objectives}

Computer simulations are limited in their representativeness, whether on product viscosity, on robot's dynamics or on human-system interactions. A prototype was developed based on the previous design stages and accounting for the results of participative simulations. It allowed to conduct new participative simulations under real conditions: such as robot inertia and payload, tank's surface reflection, product viscosity and tack, and cleaning process sewage.

The first objective of this prototype was to assess the technical feasibility of automatic cleaning cycles and to give technical specifications for the future system: speeds, forces, tools, trajectories and robot control. The second objective was to design and evaluate human-system interactions for supervisory control and teleoperation. To illustrate our approach, we focus in this section on the design and evaluation of robot teleoperation.

\section{B. Material}

Based on previous design steps, the prototype is composed of a 6-axis robot (Staubli TX90XL) ceiling mounted above a tank replica. This replica is made with the same stainless steel and surface roughness as original tanks and is composed 


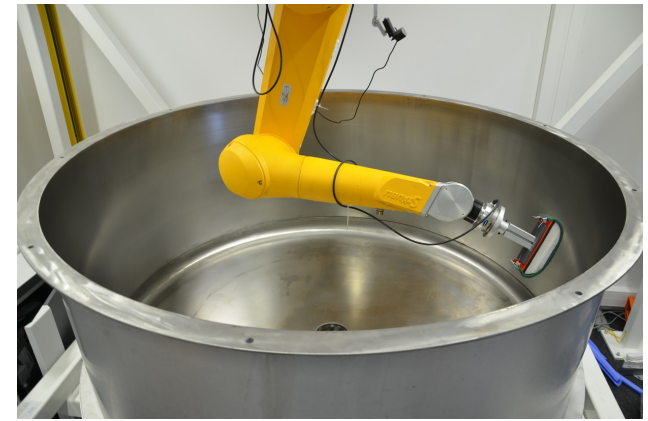

Fig. 4. Tank cleaning prototype: robot with scratching tool, placed above the tank replica.

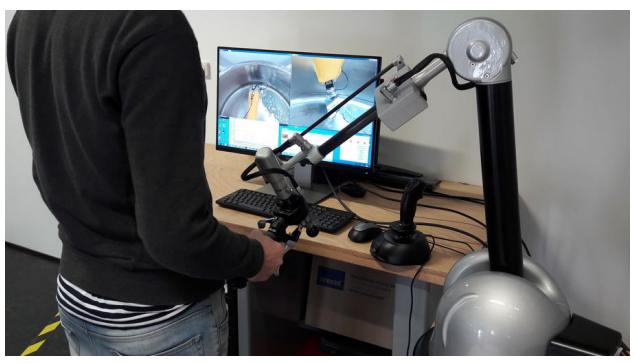

Fig. 5. Control station prototype: an operator is using the master arm to remote control the slave robot through camera views displayed on a screen.

of two parts (lateral and bottom). This prototype, set up on CEA Tech's TROPIC platform ${ }^{1}$, is illustrated on Fig. 4.

In the previous stage, operators raised concerns about joystick teleoperation. However, it was implemented on the prototype to have a more detailed assessment of it limits under real conditions. In addition, a haptic device (Haption Virtuose 6D) was implemented to test the added value of giving force feedback to the operator. Moreover, it allows to assess the number of degrees of freedom (DOF) necessary for tank cleaning. As a result of the previous design stage, two cameras were placed on the robot to provide visual feedback during remote control (Fig. 5).

For force control and for haptic teleoperation a ATI 6axis force/torque sensor is mounted on robot's end. Robot cleaning tools were designed based on current operators' manual tools. For safety reasons, pyrotechnic product could not be brought to the platform. Instead a mechanically representative inert product was used.

\section{Automatic cleaning cycles feasibility}

Scrubbing, scratching and finishing experiments were conducted to determine the feasibility of automating these cleaning stages. A position/force control was used [32] with the force applied normally to the surface and position controlled in the surface plane. We can notice that satisfying scrubbing results were obtained for a nominal substance quantity (Fig. 6). In particular, these experiments revealed that tangential speed and normal force are determining factors. Less conclusive results were obtained for scratching and

\footnotetext{
${ }^{1} \mathrm{http} / / /$ www.cea-tech.fr/cea-tech/Pages/en-regions/pfa-telerobotiqueprocedes-industriels.aspx
}

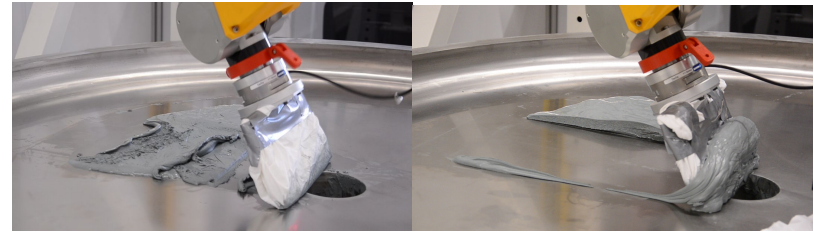

Fig. 6. Unsuccessful (left) and successful (right) automatic scrubbing.

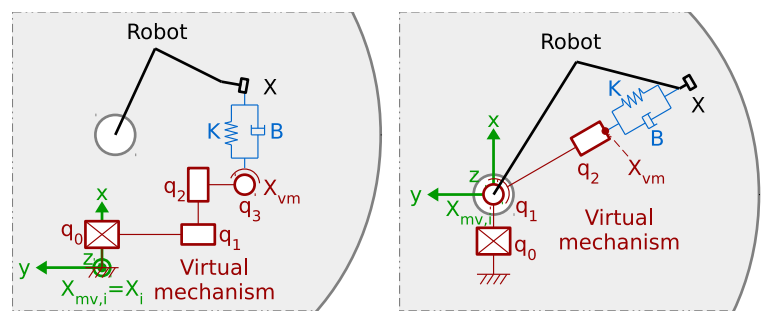

Fig. 7. Top view of virtual mechanisms displayed on a section of tank's bottom surface (4 DOF on the left, 3 DOF on the right).

finishing. At fixed speed and normal force, cleaning quality varies depending on product viscosity and tack, and on the quantity of residues left after scrubbing. These experiments confirmed the need for human expertise for these tasks.

\section{Teleoperation design}

Teleoperation must enable the operator to use his expertise to ensure cleaning quality and to handle industrial variability. As stated previously, two control devices were selected to be tested: a joystick and a haptic device. The more DOF the control device provides, the more flexibility and variety of movements are given to the operators. However, at the same time, the task execution complexity increases.

For joystick teleoperation, robot's motions were constrained to control 2 DOF, as described in Section V-D. Tool's speed was controlled with the joystick in a tangent direction to tank's surface. A controller was implemented using a virtual mechanism (VM) [32] to control tool's force normally to the surface.

On the other hand, the master arm (Haption Virtuose 6D) and the slave arm (Staubli TX90XL) are bilaterally coupled [33] to allow human's control of the force applied on the surface. Up to 6 DOF can be controlled with the haptic device. Virtual mechanisms are used to constrain motions and reduce the number of DOF. The robot end-effector is virtually coupled with a VM end-effector through a spring damper [32]. The VM forward kinematics $\left(L_{v m}\right)$ and its Jacobian define free motions, which are controlled in force, and constraints, which are controlled in position.

The design of VMs is described for the bottom surface scratching task. 2 DOF are required to control tool's position on the surface and the substance pushing force. Another DOF allows to control the distance to the surface and the normal force. 2 DOF were removed by constraining the tool's face parallelism with the tank's surface. The last DOF corresponds to tool's direction on the surface. A 4 DOF VM was implemented to let this motion free and evaluate the benefits for operators. Another VM with 3 DOF 
was implemented to constrain this motion and evaluate if operators would perform the task more efficiently. The 4 DOF VM is composed of three prismatic joints (q0, q1, q2) and a revolute joint (q3) (see the left part of Fig. 7). The 3 DOF VM is composed of two prismatic joints (q0, q2) and a revolute joint (q1) (see the right part of Fig. 7). When created, its reference position $X_{m v, i}$ is attached to tank's center. In this way, the tool is constrained to point towards the hole. Thus, the forward kinematics of the 4 DOF VM $\left(L_{v m}^{4 D O F}\right)$ and of the $3 \mathrm{DOF} \operatorname{VM}\left(L_{v m}^{3 D O F}\right)$ are:

$L_{v m}^{4 D O F}=\left\{\begin{array}{l}T x=q_{2} \\ T y=-q_{1} \\ T z=q_{0} \\ R x=0 \\ R y=0 \\ R z=q_{3}\end{array} \quad L_{v m}^{3 D O F}=\left\{\begin{array}{l}T x=q_{2} \sin \left(q_{1}\right) \\ T y=-q_{2} \cos \left(q_{1}\right) \\ T z=q_{0} \\ R x=0 \\ R y=0 \\ R z=q_{1}\end{array}\right.\right.$

Each Jacobian simply derives from the forward kinematics. Similar principles guided the design of VMs for the lateral surface cleaning. We can point out that tangential motion constraints are the same with the joystick and with the $3 \mathrm{DOF}$ VM. However, with the joystick there is no force feedback for operators and motion is speed controlled rather than position controlled.

\section{E. Participative simulations}

Simulations with operators were conducted on this prototype to evaluate supervisory control and teleoperation. In particular, joystick and haptic device control for scratching operations were compared, the added value of virtual mechanisms was studied, as well as visual feedbacks.

Once again, the purpose of the simulation, the proposed solution and the controls were explained to each operator. The simulation started with a supervisory control scenario for which the operator monitored robot automatic scrubbing through cameras. Then, teleoperated scratching tasks were performed on the bottom and lateral surface with the joystick and the haptic device (without constraint, with the 4 DOF $\mathrm{VM}$ and with the 3 DOF VM). To counterbalance learning effects, the four types of control were not used in the same order by operators. Finally, the simulation was discussed with each operator. In addition to the subjects addressed in previous simulations, the usability of each type of control was assessed through questionnaires and discussion.

Due to production constraints, only 4 operators were available to conduct simulations, one at a time. Following trends and qualitative results can be highlighted:

- The inert product is representative of the highest viscosity they have to clean, however one product chemical composition is more sticky and can be harder to remove.

- Tasks requiring to maintain tools' surface full contact with the tank are hard to perform with 6 DOF.

- For haptic device control, operators felt that the 3 DOF VM was overly constraining. The 4 DOF one is preferred, it seems more comfortable and to give sufficient freedom to adapt gestures during the cleaning.
- In the same way, operators missed a third axis on the joystick to control the tool's direction on the surface. This additional DOF would allow to execute specific trajectories that are essential on the bottom to guide the product toward the hole in some situations.

- Although the haptic device teleoperation felt more natural to operators, they considered that a 3-axis joystick without force feedback would still allow them to perform tank cleaning.

- Lightning and image quality must be studied to improve visualisation of small residues.

- An operator warned that he would be less agile with such a system and might be less efficient to clean tanks.

\section{DISCUSSION}

Several stages of design and participative simulations were presented. Computer models were used for the first and second iterations. During simulations, although these models were limited in representativeness, action scenarios and discussions allowed operators to embody the simulated operations and contribute to a more realistic descriptions of the activity [6].

The prototype allowed to conduct simulations with operators under more realistic conditions, yet it revealed that further simulations with more product and with various viscosity and tack are needed to confirm the trends. For instance, this is essential to verify the number of DOF sufficient for tank cleaning and to assess if the introduction of the system risks to decrease operations efficiency. In this way, 3-axis joystick must be implemented to be compared to haptic device teleoperation constrained with a 4 DOF VM.

In this paper, we focused on the contribution of the activity analysis and of involving operators within iterative simulations to design a human-robot manufacturing system. Thus, action scenarios, protocols and measures were not detailed at each simulation stage, but qualitative results were given to illustrate our contribution in an industrial engineering context. We highlighted the following subjects that can be assessed earlier in the design through simulations and discussions with operators: simulation's representativeness of the realworld work, interests of the proposed work activity, humanrobot system adaptation to the various contexts of use (normal, transitory, failure) and to the variability of situations, acceptability and perceived usability of the robot, situation awareness and efficiency of the human-robot system. Some inputs - such as operators regulation of variability, crystals potentially remaining, consequences of product tack, and necessary DOF for tank cleaning - were obtained thanks to the detailed activity analysis and to participative simulations. Without operators involvement, these needs and work situations would not have been identified and integrated in the early stages of the system design. Besides, we observed the increasing appropriation of the designed technical system by operators through the iterations of participative simulations. Nevertheless, it should be pointed out that the availability of operators to conduct simulations is an issue that can delay the design. 


\section{CONCLUSION AND FUTURE WORK}

We presented an approach involving robotics, ergonomics, cognitive engineering skills and operators to design of interactive robot systems preparing their introduction on industrial workstation. We illustrated the practical application of this approach on a pyrotechnic tank cleaning workstation. This paper presents the simultaneous design of an interactive robot system solution and of operator's future activity, based on participative simulations. We highlighted that involving operator is essential for several reasons:

- The introduction of an interactive robot system on an industrial workstation will have an impact on human work activity. It is the opportunity to design future work activity at the same time as the system.

- Operators provide their expert insight on how to perform the operations at best, to improve the design of the solution.

- Operators know the real-world work. These inputs are fundamental to design a system adapted to the variability of situations and to operators needs.

- It favors the future appropriation of the new system by operators.

A prototype was designed to assess remote control of a robot system as a solution for tank cleaning operations. Interaction ranging from supervisory control to teleoperation was designed and several control devices (joystick and haptic) were evaluated. Results from participative simulations conducted at each design stage were given. They revealed challenges for remote tank cleaning such as visual feedback for quality inspection, control devices usability for remote operations efficiency, and ability of the human-robot system to deal with the variability of real-world situations. The system design will be improved based on prototype results. Further simulations will be conducted to assess the solution on a wider range of situations.

Finally, our approach is currently applied to other workstations within ArianeGroup and Safran where other kinds of interactive robots are foreseen, in particular collaborative robots. Thus, we will be able to assess how it can be generalized for diverse industrial projects and to point out the specificities for various applications.

\section{REFERENCES}

[1] N. Sylla, V. Bonnet, F. Colledani, and P. Fraisse, "Ergonomic contribution of ABLE exoskeleton in automotive industry," International Journal of Industrial Ergonomics, vol. 44, no. 4, pp. 475-481, 2014.

[2] J. Krüger, T. K. Lien, and A. Verl, "Cooperation of human and machines in assembly lines," CIRP Annals - Manufacturing Technology, vol. 58, no. 2, pp. 628-646, 2009.

[3] C. Heyer, "Human-robot interaction and future industrial robotics applications," in IROS, 2010, pp. 4749-4754.

[4] L. Bainbridge, "Ironies of Automation," Automatica, vol. 19, 1983.

[5] R. Parasuraman and V. Riley, "Humans and Automation: Use, Misuse, Disuse, Abuse," Human Factors: The Journal of the Human Factors and Ergonomics Society, vol. 39, no. 2, pp. 230-253, 1997.

[6] F. Barcellini, L. Van Belleghem, and F. Daniellou, "Design projects as oppotunities for the development of activities," in Constructive Ergonomics, P. Falzon, Ed., 2014, pp. 150-163.

[7] G. V. Soska, "A Strategy for Implementation of Robotics Projects," in Handbook of Industrial Robotics, S. Y. Nof, Ed., 1999, pp. 825-828.
[8] Int. Organization for Standardization, "ISO/TR 16982: Ergonomics of human-system interaction - Usability methods supporting humancentred design," 2002.

[9] F. Daniellou, "Simulating Future Work Activity is not Only a Way of Improving Workstation Design," Activités, vol. 4, pp. 84-90, 2007.

[10] J. Harrington, Industrial cleaning technology. Kluwer Academic Publisher, 2001.

[11] C.E.B. Impianti, "Cleaning equipments." [Online]. Available: http: //www.cebimpianti.com/InfinityCEB/cms/ENG/products.html

[12] A. Shukla and H. Karki, "A review of robotics in onshore oil-gas industry," in IEEE International Conference on Mechatronics and Automation, 2013, pp. 1153-1160.

[13] S. Deng, X. Xu, C. Li, and X. Zhang, "Research on the oil tank sludge cleaning robot system," in International Conference on Mechanic Automation and Control Engineering, 2010, pp. 5938-5942.

[14] A. Pandey, A. Kaushik, A. K. Jha, and G. Kapse, "A Technological Survey on Autonomous Home Cleaning Robots," International Journal of Scientific and Research Publications, vol. 4, pp. 1-7, 2014.

[15] C. D. Jung, W. J. Chung, J. S. Ahn, M. S. Kim, G. S. Shin, and S. J. Kwon, "Optimal mechanism design of in-pipe cleaning robot," in IEEE International Conference on Mechatronics and Automation, 2011, pp. 1327-1332.

[16] Y. H. Choi, J. Y. Lee, J. D. Lee, and K. E. Lee, "SMART WINDORO V1.0: Smart window cleaning robot," in International Conference on Ubiquitous Robots and Ambient Intelligence, 2012, pp. 116-119.

[17] M. A. Goodrich and A. C. Schultz, "Human-Robot Interaction: A Survey," Foundations and Trends $®$ in Human-Computer Interaction, vol. 1, no. 3, pp. 203-275, 2007

[18] D. A. Lawrence, "Stability and Transparency in Bilateral Teleoperation," IEEE Transactions on Robotics and Automation, vol. 9, no. 5, pp. 624-637, 1993.

[19] R. Alami, et al., "Safe and dependable physical human-robot interaction in anthropic domains: State of the art and challenges," in IROS 2006.

[20] S. Haddadin, Towards Safe Robots: Approaching Asimov's 1st Law. Springer Publishing Company, 2014.

[21] Int. Organization for Standardization, "ISO 10218-2: Robots and robotic devices - Safety requirements for industrial robots - Part 2: Robot systems and integration," 2011.

[22] — "ISO/TS 15066: Robots and robotic devices - Collaborative robots," 2016

[23] V. Weistroffer, A. Paljic, P. Fuchs, O. Hugues, J. P. Chodacki, P. Ligot, and A. Morais, "Assessing the acceptability of humanrobot co-presence on assembly lines: A comparison between actual situations and their virtual reality counterparts," in IEEE International Symposium on Robot and Human Interactive Communication, 2014 pp. 377-384

[24] P. Maurice, V. Padois, Y. Measson, and P. Bidaud, "Human-oriented design of collaborative robots," International Journal of Industrial Ergonomics, vol. 57, pp. 88-102, 2017.

[25] M. M. Weeden, Failure mode and effects analysis (FMEAs) for small business owners and non-engineers: determining and preventing what can go wrong, 1952.

[26] T. B. Sheridan, Telerobotics, automation, and human supervisory control - IEEE Technology and Society Magazine. MIT Press, 1993.

[27] J. Scholtz, "Theory and evaluation of human robot interactions," in Annual Hawaii International Conference on System Sciences, 2003.

[28] E. Rohmer, S. P. N. Singh, and M. Freese, "V-REP: A versatile and scalable robot simulation framework," in IROS, 2013, pp. 1321-1326.

[29] W. Chung, L.-C. Fu, and S.-H. Hsu, "Motion Control," in Springer Handbook of Robotics, B. Siciliano and O. Khatib, Eds., 2008, pp. 133-159.

[30] A. Steinfeld, T. Fong, D. Kabe, M. Lewis, J. Scholtz, A. Schultz, and M. Goodrich, "Common Metrics for Human-Robot Interaction,' in $H R I, 2006$.

[31] M. R. Endsley and D. B. Kaber, "Level of automation effects on performance, situation awareness and workload in a dynamic control task," Ergonomics, vol. 42, no. 3, pp. 462-492, 1999.

[32] L. D. Joly, C. Andriot, and V. Hayward, "Mechanical analogies in hybrid position/force control," in IEEE International Conference on Robotics and Automation, vol. 1, 1997, pp. 835-840.

[33] L. D. Joly and C. Andriot, "Imposing motion constraints to a force reflecting telerobot through real-time simulation of a virtual mechanism," in IEEE International Conference on Robotics and Automation, vol. 1, 1995, pp. 357-362. 